\title{
ON THE THEORY OF FOURIER TRANSFORMS
}

\author{
BY EINAR HILLE AND J. D. TAMARKIN
}

1. Introduction. Let $g(s) \subset L_{2}$ over $(-\infty, \infty)$. Let

$$
G(u ; a)=(2 \pi)^{-1 / 2} \int_{-a}^{a} e^{-i u s} g(s) d s .
$$

According to the classical result of the Plancherel theory of Fourier transforms, $G(u ; a)$ tends in the mean of order 2 to a function $G(u) \subset L_{2}$ as $a \rightarrow \infty$. This function is designated as the Fourier transform (in $L_{2}$ ) of $g(s)$. We shall write

$$
T\{u ; g(s)\}=G(u)=\underset{a \rightarrow \infty}{\lim } G(u ; a) .
$$

The functions $g(s)$ and $G(u)$ are reciprocal in the sense that

$$
g(s)=T\{s ; G(-u)\}
$$

which means that

$$
g(s)=\underset{a \rightarrow \infty}{\operatorname{li} . m} g(s ; a) ; \quad g(s ; a)=(2 \pi)^{-1 / 2} \int_{-a}^{a} e^{i u s} G(u) d u .
$$

As an immediate consequence of the convergence in the mean of $G(u ; a)$ and $g(s ; a)$, we have, almost everywhere,

$$
\begin{aligned}
G(u) & =(2 \pi)^{-1 / 2} \frac{d}{d u} \int_{-\infty}^{\infty} g(s) \frac{1-e^{-i s t}}{i s} d s, \\
g(s) & =(2 \pi)^{-1 / 2} \frac{d}{d s} \int_{-\infty}^{\infty} G(u) \frac{1-e^{i u s}}{-i u} d u .
\end{aligned}
$$

The reciprocity between $g$ and $G$ is expressed here in terms not involving the convergence in the mean.

Assume now that $g(s) \subset L_{p}, 1<p<2$. Denote by $p^{\prime}$ the conjugate exponent, $p^{\prime}=p /(p-1), 1 / p+1 / p^{\prime}=1$. Titchmarsh* showed that Plancherel's theory can be extended, at least in part, to the present case. Indeed he proved that $G(u ; a)$ con-

* E. C. Titchmarsh, A contribution to the theory of Fourier transforms, Proceedings of the London Mathematical Society, (2), vol. 23 (1925), pp. 279-289. We have slightly modified Titchmarsh's notation inasmuch as he deals with cosine- and sine-transforms, while we use exponential transforms. 
verges in the mean of order $p^{\prime}$ to a function $G(u) \subset L_{p^{\prime}}$, which satisfies the same reciprocity relations (4), (5). Titchmarsh did not prove, however, that, conversely, (3) holds. On the basis of the material developed by Titchmarsh we do not know whether $g(s ; a)$ tends in the mean of order $p$ to $g(s)$; hence we do not know whether $g(s)$ is the Fourier transform (in $L_{p}$ ) of $G(-u)$. A scrutiny of the literature reveals the unexpected fact that this question has never been investigated, ${ }^{*}$ which, in our opinion, represents an undesirable gap in the theory of Fourier transforms. The purpose of the present note is to fill in this gap and to prove (3) in the case $1<p \leqq 2$. An analogy between the theory of Fourier transforms and that of Fourier series should be pointed out here. We may consider the Fourier transform $G(u)$ of $g(s)$ as an analog of the sequence of Fourier coefficients $\left\{G_{n}\right\}$ of $g(s)$ (in the case where $g$ is periodic and is being expanded in Fourier series). Then $g(s ; a)$ appears as an analog of the $n$th partial sum $s_{n}(s ; g)$ of $g(s)$ which is known to converge to $g(s)$ in the mean of order $p$. This analogy goes even further. It is known that if $g(s) \subset L_{1}$, over $(-\pi, \pi), s_{n}(s ; g)$ does not necessarily converge in the mean of order 1 to $g(s)$. $\dagger$ We shall prove that this is also the case for the Fourier transforms.

It will be essential for our discussion to consider the class of functions $\subset L_{p}$ over $(-\infty, \infty)$ as a linear vector metric complete space with the metric defined by

$$
\|g\|_{p}=\left[\int_{-\infty}^{\infty}|g(s)|^{p} d s\right]^{1 / p} .
$$

By $R^{p}$ we shall mean the limit in the mean of order $p$. Some known properties of linear transformations of such spaces will be used in the sequel.t

* Contrary to our unfounded statement in a recent paper. See E. Hille and J. D. Tamarkin, On the summability of Fourier series, III, Mathematische Annalen, vol. 108 (1933), pp. 525-577 (p. 530). According to results of the present note, however, this statement is correct.

$\dagger$ The case $p=1$ goes back to H. Hahn, Ueber die Darstellung gegebener Funktionen durch singuläre Intergrale, II, Denkschriften Wierer Akademie, Math.-Nat. Kl., vol. 93 (1916), p. 557-692 (p. 681). For $p>1$ see M. Riesz, Sur les fonctions conjuguées, Mathematische Zeitschrift, vol. 27 (1927), pp. 218-244 (p. 230). M. Riesz states at the end of his paper that to his results concerning Fourier series there correspond similar results for Fourier integrals.

$\ddagger$ We refer for these properties to the recent book by S. Banach, Théorie des Opérations Linéaires, Warsaw, 1932. 
2. The Case $1<p \leqq 2$. If $g(s) \subset L_{p}, 1<p \leqq 2$, we have

$$
G(u)=\underset{a \rightarrow \infty}{R^{p^{\prime}}}(2 \pi)^{-1 / 2} \int_{-a}^{a} g(t) e^{-i u t} d t .
$$

Here we may multiply by any function $\subset L_{p}$ and integrate under the integral sign. Thus

$$
\begin{aligned}
g(s ; a) & =(2 \pi)^{-1 / 2} \int_{-a}^{a} G(u) e^{i s u} d u \\
& =(2 \pi)^{-1} \int_{-\infty}^{\infty} g(t) d t \int_{-a}^{a} e^{i u(s-t)} d u \\
& =\frac{1}{\pi} \int_{-\infty}^{\infty} g(t) \frac{\sin a(s-t)}{s-t} d t .
\end{aligned}
$$

Let $f(s)$ be any function $c L_{p}$. The integral

$$
\tilde{f}(s)=\frac{1}{\pi} \int_{-\infty}^{\infty} \frac{f(t)}{s-t} d t
$$

exists (in the sense of Cauchy's principal value at $t=s$ ) almost everywhere and is designated as the function conjugate to $f(s)$.

Moreover, $\tilde{f}(s) \subset L_{p}$ whenever $f(s) \subset L_{p}$. Furthermore, there exists a positive constant $M_{p}$ depending only on $p$ and such that*

$$
\|\tilde{f}\|_{p} \leqq M_{p}\|f\|_{p}
$$

Upon introducing the functions

$$
g_{a}^{\prime}(t)=g(t) \cos a t, \quad g_{a}^{\prime \prime}(t)=-g(t) \sin a t
$$

and their conjugates $\tilde{g}_{a}^{\prime}(s), \tilde{g}_{a}^{\prime \prime}(s), \dagger$ we may rewrite (6) in the form

By (7),

$$
g(s ; a)=\tilde{g}_{a}^{\prime}(s) \sin a s+\tilde{g}_{a}^{\prime \prime}(s) \cos a s .
$$

$$
\left\|\tilde{g}_{a}^{\prime}\right\|_{p} \leqq M_{p}\|g(t) \cos a t\|_{p} \leqq M_{p}\|g\|_{p},\left\|\tilde{g}_{a}{ }^{\prime \prime}\right\|_{p} \leqq M_{p}\|g\|_{p} .
$$

* M. Riesz, loc. cit., p. 234.

$\dagger$ For the analogous procedure in the case of Fourier series, see M. Riesz, loc. cit., p. 230, and A. Kolmogoroff, Sur les fonctions harmoniques conjuguées et sur les séries de Fourier, Fundamenta Mathematicae, vol. 7 (1925), pp. 23-28. 
Hence

$$
\|g(s ; a)\|_{p} \leqq\left\|\tilde{g}_{a}^{\prime}\right\|_{p}+\left\|\tilde{g}_{a}^{\prime \prime}\right\|_{p} \leqq 2 M_{p}\|g\|_{p} .
$$

For each $a$, the expression $g(s ; a)$ constitutes as a linear transformation on $L_{p}$ to $L_{p}$, and (8) shows that the family of these transformations, obtained when $a$ varies, is uniformly limited.

Now assume that $g(s)$ is a step function of a finite number of steps, vanishing outside of a finite interval $(-N, N)$. For such a function we shall prove directly that

$$
\|g(s ; a)-g(s)\|_{p} \rightarrow 0 \text { as } a \rightarrow \infty .
$$

Since our function $g(s)$ is a linear combination of step functions defined by

$$
g(s)=\left\{\begin{array}{l}
1 \text { if } \alpha \leqq s \leqq \beta, \\
0 \text { outside }(\alpha, \beta),
\end{array} \quad(-N \leqq \alpha<\beta \leqq N),\right.
$$

it will be sufficient to prove our assertion for a function of this type. For such a function, however,

$$
g(s ; a)=\int_{\alpha}^{\beta} \frac{\sin a(s-t)}{s-t} d t .
$$

It is readily seen that

$$
g(s ; a)=O\left[(s-N)^{-1} a^{-1}\right], \quad(s \geqq 2 N) .
$$

Since $g(s)=0$ for $|s|>N$, we have

$$
\int_{2 N}^{\infty}|g(s)-g(s ; a)|^{p} d s=O\left[a^{-p} \int_{2 N}^{\infty} \frac{d s}{(s-N)^{p}}\right]=O\left(a^{-p}\right)
$$

as $a \rightarrow \infty$. Similarly

$$
\int_{-\infty}^{-2 N}|g(s)-g(s ; a)|^{p} d s=O\left(a^{-p}\right) .
$$

On the other hand the integral

$$
\frac{1}{\pi} \int_{\alpha}^{\beta} \frac{\sin a(s-t)}{s-t} d t=\frac{1}{\pi} \int_{-2 N}^{2 N} g^{*}(t) \frac{\sin a(s-t)}{s-t} d t
$$

may be considered as the classical Dirichlet integral of the function $g^{*}(s)$ which is periodic, of period $4 N$, and coincides with $g(s)$ in the interval $(-2 N, 2 N)$. It is well known from the theory 
of Fourier series, and also can be proved directly, that this Dirichlet integral converges to $g^{*}(s)$ in the mean of order $p$, over the interval $(-2 N, 2 N)$. Hence

$$
\begin{aligned}
& \int_{-2 N}^{2 N}|g(s)-g(s ; a)|^{p} d s \\
= & \int_{-2 N}^{2 N}\left|g^{*}(s)-g(s ; a)\right|^{p} d s \rightarrow 0 \text { as } a \rightarrow \infty .
\end{aligned}
$$

Consequently

$$
\int_{-\infty}^{\infty}|g(s)-g(s ; a)|^{p} d s=\int_{-2 N}^{2 N} \cdots+\int_{-\infty}^{-2 N} \cdots+\int_{2 N}^{\infty} \cdots \rightarrow 0
$$

as $a \rightarrow \infty$, which is the desired result.

Since the family of transformations represented by $g(s ; a)$ is uniformly limited, and since the step-functions constitute a dense sub-set of $L_{p}$, it follows immediately that

$$
\|g(s)-g(s ; a)\|_{p} \rightarrow 0 \text { as } a \rightarrow \infty,
$$

for an arbitrary function $g(s) \subset L_{p}$. We may therefore state the following addition to the results of Titchmarsh mentioned above.

THEOREM. If $g(s) \subset L_{p}, 1<p \leqq 2$, and if

$$
G(u)=\underset{a \rightarrow \infty}{R^{p^{\prime}}}(2 \pi)^{-1 / 2} \int_{-a}^{a} g(s) e^{-i u s} d s
$$

is the Fourier transform (in $L_{p^{\prime}}$ ) of $g(s)$, then, conversely, $g(s)$ is the Fourier transform (in $L_{p}$ ) of $G(-u)$, and we have

$$
g(s)=\underset{a \rightarrow \infty}{\ell^{p}}(2 \pi)^{-1 / 2} \int_{-a}^{a} G(u) e^{i u s} d u .
$$

3. The Case $p=1$. We now proceed to show that our theorem fails in the case $p=1$. To do this we have to exhibit an example of a function $g(s) \subset L_{1}$, such that, if we set

$$
\begin{gathered}
G(u)=(2 \pi)^{-1 / 2} \int_{-\infty}^{\infty} g(s) e^{-i u s} d s, \\
g(s ; a)=(2 \pi)^{-1 / 2} \int_{-a}^{a} G(u) e^{i u s} d u,
\end{gathered}
$$

the function $g(s ; a)$ does not converge to $g(s)$ in the mean of or- 
der 1 . The example which we are going to construct will show even more, namely, that the corresponding $g(s ; a)$ does not even belong to $L_{1}$.

In constructing the example in question we are again guided by the analogy with Fourier series. It is well known* that the series

$$
f(x)=\sum_{n=2}^{\infty} \frac{\cos n x}{\log n}
$$

is a Fourier series of $f(x) \subset L_{1}$, but that its partial sums $s_{p}(x ; f)$ do not converge to $f(x)$ in the mean of order 1 over $(-\pi, \pi)$. We now put

$$
g(s)=(2 \pi)^{-1 / 2} \int_{0}^{\infty} \frac{\cos u s}{\log (2+u)} d u .
$$

It is plain that this integral converges uniformly over every interval, finite or not, which is separated from the point $s=0$. Moreover, it is easy to show that $g(s)>0$ for $s \neq 0$. It suffices to consider positive values of $s$ only. Let $s>0$ be fixed. We put

$$
q(u)=(2+u) \log ^{2}(2+u), \quad \delta_{\nu}=\nu \pi / s, \quad(\nu=0,1,2, \cdots) .
$$

Then, on integrating by parts in the right-hand member of (9), we see that

$$
(2 \pi)^{1 / 2} g(s)=\frac{1}{s} \int_{0}^{\infty} \frac{\sin u s}{q(u)} d u=\sum_{\nu=0}^{\infty} \frac{1}{s} \int_{\delta_{\nu}}^{\delta_{\nu+1}} \frac{\sin u s}{q(u)} d u .
$$

Put

Hence

$$
I_{\nu}(s)=\frac{1}{s} \int_{\delta \nu}^{\delta_{\nu+1}} \frac{\sin u s}{q(u)} d u=(-1)^{\nu} s^{-2} \int_{0}^{\pi} \frac{\sin \tau d \tau}{q(\nu \pi+\tau / s)} .
$$

$$
\left|I_{\nu}\right|<I_{\nu-1}||, \quad(\nu=1,2, \cdots),
$$

and $\sum_{0}^{\infty} I_{\nu}$ appears as an alternating series in which the absolute value of the general term approaches zero and the first term is positive. The sum of such a series is positive. Hence $g(s)>0$. An integration by parts applied to the middle term in (10) shows that, for large values of $s, g(s)=O\left(s^{-2}\right)$. This ensures the integrability of $g(s)$ over every interval $(\epsilon, \infty), \epsilon>0$. Since $g(s)>0$, to

* A. Kolmogoroff, Sur l'ordre de grandeur de coefficients de la série de Fourier, Bullétin International de l'Académie Polonaise, Classe de Sciences mathématiques, 1923, pp. 83-86. See also E. Hille and J. D. Tamarkin, On the summability of Fourier series, II, Annals of Mathematics, (2), vol. 34 (1933), pp. 329-348 (pp. 347-348). 
establish the integrability of $g(s)$ over $(0, \epsilon)$ it suffices to prove that, for any fixed positive $\alpha$,

$$
\int_{e}^{\alpha} g(s) d s
$$

is bounded as $\epsilon \rightarrow 0$. In view of the uniform convergence of the integral in (9) over $(\epsilon, 1)$ we have

$$
\begin{aligned}
\int_{\epsilon}^{1} g(s) d s & =(2 \pi)^{-1 / 2} \int_{0}^{\infty} \frac{d u}{\log (2+u)} \int_{\epsilon}^{1} \cos u s d s \\
& =(2 \pi)^{-1 / 2}\left\{\int_{0}^{\infty} \frac{\sin u d u}{u \log (2+u)}-\int_{0}^{\infty} \frac{\sin u d u}{u \log (2+u / \epsilon)}\right\} .
\end{aligned}
$$

A simple application of the second law of the mean shows that the last integral tends to zero with $\epsilon$. Thus we see that $g(s)$ is integrable over $(0, \infty)$, and, being even, it is integrable over $(-\infty, \infty)$. By the uniqueness theorem of Fourier integrals, it is plain that

$$
G(u)=[\log (2+|u|)]^{-1}=(2 \pi)^{-1 / 2} \int_{0}^{\infty} g(s) \cos s u d s
$$

is the Fourier transform of $g(s)$ in $L_{\infty}$, that is, $G(u ; a)$ converges uniformly to $G(u)$ as $a \rightarrow \infty$. Now compute

$g(s ; a)=(2 \pi)^{-1 / 2} \int_{0}^{a} G(u) \cos s u d u=(2 \pi)^{-1 / 2} \int_{0}^{a} \frac{\cos s u d u}{\log (2+u)}$.

An integration by parts shows that

$$
(2 \pi)^{1 / 2} g(s ; a)=\frac{\sin s a}{s \log (2+a)}+\frac{1}{s} \int_{0}^{a} \frac{\sin s u}{q(u)} d u .
$$

As before, we see that the second term of the right-hand member is $O\left(s^{-2}\right)$ for $s \rightarrow \infty$, and since the first term is not absolutely integrable over $(0, \infty)$ it results that $g(s ; a)$ does not belong to $L_{1}$.

Brown UnIVERsity and YaLe University 